\title{
REVISIÓN Y ADAPTACIÓN DEL CONCEPTO "INNOVACIÓN" AL CONTEXTO EMPRESARIAL ESPAÑOL
}

\section{REVIEW AND ADAPTATION OF "INNOVATION" IN THE SPANISH BUSINESS CONTEXT}

Platero Jaime, Manuel (Universidad Europea de Madrid) ${ }^{*}$

\section{RESUMEN}

La innovación se ha convertido en una realidad innegable en el contexto de la gestión de la empresa, independientemente de su tamaño o actividad. En las últimas décadas, numerosas investigaciones han estudiado la capacidad innovadora de la empresa, sus consecuencias macroeconómicas y la relación que guarda la innovación con ciertos factores macros y micros. En este sentido, algunos trabajos han analizado la influencia de determinados factores en la capacidad innovadora de las empresas, prestando especial atención a la de gran tamaño y descuidando en ocasiones el análisis en el ámbito de las empresas de estructuras más reducidas que suelen tener otro tipo de recursos y capacidades, muy diferentes a los que representan a las más grandes. Precisamente este trabajo pretende contribuir a mejorar el conocimiento sobre la innovación empresarial en entidades organizativas de tamaño más limitado, que son las que más peso tienen en la mayoría de las economías emergentes y desarrolladas. Este trabajo pretende estudiar y evaluar la evolución que presenta el concepto "innovación", para redefinirlo y adaptarlo a las características intrínsecas de la microempresa española. Con ello, se pretende contribuir a mejorar el conocimiento sobre la innovación y sus formas en el contexto de las microempresas de España y sentar las bases conceptuales para futuras investigaciones en el ámbito de las pequeñas empresas.

Palabras claves: Innovación, microempresa, recursos y capacidades, competitividad. JEL: L25, M20, O31.

\begin{abstract}
Innovation has become an undeniable reality in the management of the firm, regardless of size or activity. In recent decades, numerous studies have examined the innovative capacity of the company, its macroeconomic consequences and the relationship of innovation with certain macro and micro factors. In this sense, some studies have analysed the influence of certain factors on the innovative capacity of enterprises, paying special attention to large business and sometimes neglecting the analysis in the area of smaller companies. Precisely this work tries to improve the knowledge about business innovation in organizational entities of more limited size, which are those that have more weight in most emerging and developed economies. This work aims to study and analyse the evolution of the concept "innovation" in order to redefine and adapt this concept to the intrinsic characteristics of the Spanish microenterprise. This study try to improve the understanding of innovation and its forms in the Spanish context, and lay the conceptual foundation for future research in the field of small businesses.
\end{abstract}

Key words: Innovation, microenterprise, resources and capabilities, competitiveness. JEL: L25, M20, O31.

* Departamento de Economía y Finanzas, C/ Tajo s/n. Urb. El Bosque, 28670, Villaviciosa de Odón, Madrid. manuel.platero@uem.es

Recibido: Septiembre de 2015. Aceptado: Octubre de 2015. 


\section{INTRODUCCIÓN}

La globalización es, hoy por hoy, una realidad en el actual paradigma empresarial. El fomento de la innovación y la iniciativa empresarial resultan determinantes para entender el nivel de competitividad de las regiones (Platero-Jaime, 2014). Tradicionalmente, los esfuerzos para explicar la competitividad de una región se han basado en una perspectiva global, concentrándose en las características de los factores involucrados en las políticas gubernamentales y en los indicadores macroeconómicos. El mercado actual, caracterizado por una continua saturación de la demanda, ha obligado a las empresas a considerar la innovación como uno de los principales objetivos empresariales conjuntamente con la mejora de la productividad. Por ende, el análisis y la gestión de la innovación a nivel macroeconómico pero también microeconómico, resultan esenciales dados los niveles de competitividad empresarial de nuestros días.

El presente trabajo pretende contribuir a mejorar el conocimiento sobre la innovación empresarial en entidades organizativas de tamaño más limitado, que son las que más peso tienen en la mayoría de las economías emergentes y desarrolladas. Este trabajo pretende estudiar y evaluar la evolución que presenta el concepto "innovación", para redefinirlo y adaptarlo a las características intrínsecas de la microempresa española.

El estudio se inicia con una breve revisión de la literatura vinculada al concepto de la innovación en términos de competitividad y estrategia para posteriormente, comparar las principales diferencias que definen la naturaleza y las capacidades de la pequeña y la gran empresa. A continuación se justifica la importancia y el papel que juega la microempresa en las economías actuales y seguidamente se realiza un análisis conceptual sobre la evolución que ha presentado el término innovación en las últimas décadas. Finalmente, se obtienen unas conclusiones que se espera puedan ayudar a sentar las bases conceptuales del concepto innovación en el ámbito de la microempresa española.

\section{LA INNOVACIÓN COMO CONCEPTO DIFERENCIAL ENTRE LA GRAN Y LA PEQUEÑA EMPRESA}

Desde el punto de vista macroeconómico, Porter (1990, 1996) propone un nuevo paradigma de competitividad basado en el proceso de innovación dinámica de las empresas y las industrias, a través del cual las interrelaciones entre empresas, instituciones y mercados propician el desarrollo competitivo de las regiones. Diversos autores llevaron a cabo nuevas aportaciones en relación con los elementos contextuales y relacionales del proceso de innovación que contribuyen al desarrollo económico regional (Zahra et al., 1988; Roberts y Amit, 2003). En este sentido, la innovación se ha comenzado a considerar como un factor crucial en la competitividad empresarial y, a raíz de ello, se empiezan a estudiar factores que favorecen y limitan la capacidad de innovación de las empresas (Broekel y Brenner, 2011; Morales et al., 2013).

En este sentido, Mogollón y Vaquero (2004) afirman que los esfuerzos de innovación de las empresas son la prueba fehaciente de la creciente concienciación de éstas por gestionar la innovación y alcanzar así la ventaja competitiva (Baker y Sinkula, 1999; Andriopoulos y Lewis, 2010). La relación entre la innovación, la ventaja competitiva y el crecimiento empresarial ha sido ampliamente analizada por la literatura (Hashi y Stojčić, 2013; Camisón y Villar-López, 2014).

Para que una organización optimice su capacidad innovadora de forma sostenible debe de incorporar la innovación como un componente significativo en su estrategia, además de disponer de los suficientes recursos para el desarrollo y el lanzamiento de nuevos productos, y 
tener la suficiente flexibilidad en su estructura y sus procesos para resolver los problemas del día a día de forma creativa, siendo capaz de conectar la capacidad innovadora con los objetivos generales y específicos (Bhaskaran, 2006; Hutter et al., 2013).

La innovación se ha consolidado como uno de los factores estratégicos claves de las empresas, principalmente porque constituye el principal medio a través del cual se puede identificar y explotar nuevas oportunidades de negocio (Kunz et al., 2011; Laforet, 2013). Schumpeter (1934), a través de varias de sus obras como "The Theory of Economic Development" o "Capitalism, Socialism and Democracy", fue uno de los primeros autores en exponer el papel de la innovación en los ámbitos estratégico, empresarial y económico. En la primera de las obras citadas, el autor propone y estudia como la innovación resulta esencial en el ciclo de vida empresarial, a través de la teoría de la "destrucción creativa", la cual describe como los nuevos productos destruyen viejas empresas y modelos de negocio que dan paso a nuevos formatos de mercado. Sin embargo, el autor también plantea, a través de la segunda obra anteriormente citada, como el énfasis de la innovación cambia hacia los departamentos de I+D de las grandes empresas. Estas consideraciones sobre la innovación dan pie a la reflexión y a numerosas investigaciones durante las siguientes décadas y hasta el día de hoy, y resultan esenciales para el desarrollo del presente trabajo. La innovación es considerada por muchos investigadores como un factor crítico de la empresa para competir eficazmente en los mercados nacionales y mundiales (Hitt et al., 2001). Hamel (2000) sostiene que la innovación es el componente más importante en la estrategia de una empresa.

La literatura considera que la innovación es uno de los factores claves del éxito de la empresa a largo plazo (Simon, 2009; Kmieciak et al., 2012), y argumenta cómo las empresas con capacidad para innovar podrán responder a los cambios del entorno mejor y más rápidamente que las empresas no innovadoras (Romero y Martínez-Román, 2012).

Según Güngör y Gözlu (2012), la innovación se ha convertido en una realidad innegable en el contexto de la gestión de la empresa, independientemente de su tamaño o actividad. Es por ello que la capacidad de innovación está en la agenda de muchos investigadores. Como resultado, hay una gran cantidad de estudios que se han llevado a cabo en esta materia. Sin embargo, los resultados no son concluyentes y con frecuencia resultan contradictorios, hecho que demuestra la necesidad de dedicar más esfuerzos de investigación en este área. Hay muchos factores identificados en las publicaciones científicas que influyen en la capacidad de innovación de las empresas (Love y Roper, 2001; Rowley et al., 2011). Sin embargo, existen diferencias contrastables entre los factores determinantes de la capacidad innovadora en el ámbito de las grandes y pequeñas empresas (Koski et al., 2009). Precisamente, varios trabajos ya proponían estudiar los factores que influyen en el carácter innovador de la microempresa española y del propio microempresario, para contribuir así a mejorar el conocimiento sobre la innovación empresarial en entidades organizativas de tamaño más limitado, que son las que más peso tienen en la mayoría de las economías emergentes y desarrolladas (Boumediene et al., 2009; Nieto y Santamaría, 2010).

En este sentido y según lo expuesto en los párrafos anteriores, el tamaño de la empresa no parece ser un factor determinante en la necesidad de innovar para lograr la subsistencia de la misma, sin embargo, el tamaño sí que puede influir en cómo innovar o sobre los factores que influyen en la capacidad innovadora de la empresa (Laforet, 2013). Una de las características más concluyentes del presente panorama internacional es que la preeminencia de las grandes dimensiones empresariales y productivas como instrumento de generación de economías de escala, ha abierto paso en las coordenadas económicas actuales a procesos de difusión y descentralización de los procesos de producción, en los cuales encuentran un hueco 
adecuado y competitivo las pequeñas unidades empresariales (Bednarzik, 2000; Hutter et al., 2013).

La consecuencia del actual contexto empresarial global, convierte en requisito fundamental estudiar en profundidad aquellas diferencias preeminentes entre las grandes y pequeñas empresas, en términos de comportamientos empresariales, competitividad e innovación (Audretsch y Lehmann, 2005; Booyens, 2011).

Desde mediados de los ochenta, las redes regionales de pequeñas empresas comenzaron a surgir amenazando la hegemonía de la producción en masa de las grandes corporaciones industriales. Este cambio de paradigma condujo a una nueva creencia sobre la viabilidad económica de la producción en pequeña escala y su capacidad de contribuir, no sólo a la creación de empleo y riqueza, sino a una nueva forma de entender y estudiar la productividad y la competitividad a través de la innovación (Wennekers et al., 2005; Audretsch, 2012).

Existen grandes diferencias en la gestión de la innovación entre las pequeñas y las grandes empresas y, por supuesto, en los resultados derivados de ésta (Laforet, 2013). Las grandes empresas tienden a tener una estructura más burocrática, con un mayor número de niveles de la organización y suelen contar con un departamento propio de $\mathrm{I}+\mathrm{D}$, no como en el caso de las pequeñas. Por lo tanto, la integración del proceso de innovación en los diferentes niveles de las grandes empresas es en ocasiones compleja, todo lo contrario que en las pequeñas empresas (Koski et al., 2009).

Por todo ello, resulta interesante plantear las bases de un nuevo marco de estudio que responda a las necesidades funcionales y conceptuales de la gestión de la innovación en el ámbito de la microempresa.

El presente artículo se adscribe, por tanto, a una línea de investigación que trata de explicar las principales diferencias entre los factores que determinan la innovación en las empresas grandes y en las pequeñas (Andersson y Lööf, 2011; Hutter et al., 2013). Así pues, este trabajo pretende aportar nuevas contribuciones al estudio de los factores determinantes de la innovación en las pequeñas empresas y, en concreto, en las microempresas (Booyens, 2011).

\section{LA IMPORTANCIA DE LA MICROEMPRESA}

Resulta vital comprender el papel real que juegan las microempresas en los actuales sistemas económicos de nuestros días. Para ello, a continuación se define la microempresa y se fundamenta su impacto socioeconómico en España y el mundo.

Según la Comisión Europea (2013a), las pequeñas y medianas empresas (PYMES) pueden definirse en términos del número de empleados y en términos de volumen de negocios o balance general anual como se muestra en el cuadro 1:

\section{CUADRO 1: CATEGORÍAS DE EMPRESAS SEGÚN DEFINICIÓN Y REQUISITOS ${ }^{1}$.}

\begin{tabular}{|c|c|c|c|}
\hline Categoría de Empresa & Empleados & Volumen de negocios & Balance Anual \\
\hline Micro & $<10$ & $<€ 2$ millones & $<€ 2$ millones \\
\hline Pequeña & $<50$ & $<€ 10$ millones & $<€ 10$ millones \\
\hline Mediana & $<250$ & $<€ 50$ millones & $<€ 43$ millones \\
\hline
\end{tabular}

Fuente: Comisión Europea (2008)

\footnotetext{
${ }^{1}$ Según se define en la legislación comunitaria: recomendación de la UE2003/361. Las categorías de tamaño empleadas en este informe siguen las definiciones de la base de datos de estadísticas empresariales Eurostat.
} 
El presente trabajo considera, de acuerdo con la legislación comunitaria (recomendación de la UE 2003/361), la definición de las diferentes categorías de empresas según el número de empleados. Tal y como establece la base de datos "Eurostat Structural Business Statistics", dichas categorías son: microempresas (0-9 personas ocupadas), pequeñas empresas (10-49 personas empleadas), empresas medianas ( 50 a 250 personas trabajadores por cuenta propia) y grandes empresas (250 o más personas empleadas) (Reporte Anual sobre PYMES en Europa 2012/2013 “Una recuperación en el horizonte", 2013).

En este sentido, la Comisión Europea, publicó la nueva definición de microempresa, vigente desde 2005, basándose en el artículo dos de la recomendación de la Comisión de 6 de mayo de 2003. Según dicha definición se considera microempresa aquella empresa que tienen menos de diez trabajadores, un límite de dos millones de euros en balance general y un límite de dos millones de euros para la cifra de negocios

En España, el Real Decreto 1515/2007, de 16 de noviembre, por el que se aprueba el Plan General de Contabilidad de Pequeñas y Medianas Empresas y los criterios contables específicos para microempresas, define esta, como la pequeña empresa que durante dos ejercicios económicos consecutivos, reúnan, a la finalización de cada uno de ellos, al menos, dos de las siguientes características:

- Número medio de trabajadores, no superior a diez.

- Importe neto de la cifra anual de negocios, no superior a dos millones de euros.

- Total de partidas del Activo de su Balance, no superior a un millón de euros.

El papel de la microempresa en numerosos países del mundo y, en concreto, en España es y seguirá siendo fundamental a la hora de explicar el comportamiento y el desarrollo de los sistemas económicos locales. Por ende, es necesario destacar la importancia del estudio del comportamiento y el funcionamiento de esta unidad empresarial que cada día resulta más determinante en las relaciones comerciales y socioeconómicas en todo el mundo.

Un primer motivo que justifica la relevancia de la figura de la microempresa en las diferentes economías del mundo, no es otro que su representatividad en la estructura empresarial de cada país. Por ejemplo, en España y en Europa, en el año en el que comienza la presente investigación (2010), la distribución de empresas según el número de asalariados era la que se presenta en el cuadro 2:

CUADRO 2: EMPRESAS SEGÚN ESTRATO DE ASALARIADOS Y
PORCENTAJE TOTAL, EN ESPAÑA Y EN LA UE-27 (2010)
\begin{tabular}{|c|c|c|c|c|c|c|}
\hline \multicolumn{1}{|c|}{} & $\begin{array}{c}\text { Micro } \\
\mathbf{0 - 9}\end{array}$ & $\begin{array}{c}\text { Pequeñas } \\
\mathbf{1 0 - 4 9}\end{array}$ & $\begin{array}{c}\text { Medianas } \\
\mathbf{5 0 - 2 4 9}\end{array}$ & $\begin{array}{c}\text { PYME } \\
\mathbf{0 - 2 4 9}\end{array}$ & $\begin{array}{c}\text { Grandes } \\
\mathbf{2 5 0} \text { y más }\end{array}$ & Total \\
\hline \multirow{2}{*}{$\begin{array}{c}\text { ESPAÑA } \\
\%\end{array}$} & 3.091 .849 & 130.448 & 20.888 & 3.243 .185 & 3.801 & 3.246 .986 \\
\cline { 2 - 7 } & 95,2 & 4,0 & 0,6 & 99,9 & 0,1 & 100 \\
\hline UE-271\% & 92,1 & 6,6 & 1,1 & 99,8 & 0,2 & 100 \\
\hline
\end{tabular}

Fuente: INE, DIRCE 2011 (datos a 1 de enero de 2011) y Comisión Europea, "Annual Report on EU Small and Medium sized Enterprises 2010/2011". Estimaciones para 2010 
En concreto, en el caso español y según datos más actuales del Instituto Nacional de Estadística (INE), Directorio Central de Empresas (DIRCE), referidos al año 2013²:

- El 99,9 por 100 del tejido empresarial español estaba constituido por empresas de menos de doscientos cincuenta asalariados; siguiendo las definiciones de la Comisión Europea serían PYMES.

- Más del 99,3 por 100 del tejido empresarial español estaba constituido por empresas de menos de cincuenta asalariados, siguiendo las definiciones de la Comisión Europea serían pequeñas empresas.

- El 95,7 por 100 del tejido empresarial español estaba constituido por empresas de menos de diez asalariados, es decir, siguiendo las indicaciones de las definiciones de la Comisión Europea, por microempresas.

Aunque la distribución de la estructura empresarial de la UE de los 27 y de España, son muy similares, lo cierto es que si el tejido empresarial europeo es claramente intensivo en microempresas, el contexto español es si cabe incluso más intensivo que el del conjunto de la Unión. Así lo demuestran los datos publicados por el DIRCE de 2013, los cuales revelan como el conjunto de países de la UE-27 tienen un 92,1 por 100 de microempresas, mientras que España alcanza el 95,7 por 100. De cualquier forma, estos datos manifiestan una clara preeminencia de la figura de la microempresa en el tejido empresarial europeo, con lo que ello supone en términos sociales y económicos.

Como se puede observar la evolución de la representatividad de la microempresa en el tejido empresarial español no supone cambios significativos, apreciándose un leve incremento en el año 2013 (95,7 por 100) respecto al año 2010 (95,2 por 100), pero siendo prácticamente despreciable (0,5 por 100). Por el contrario, esta misma evolución en los mismos años de observación para el caso europeo, no supone cambio alguno (92,1 por 100). Así pues, se puede afirmar que la estructura empresarial española y europea es y sigue siendo intensiva en microempresas, con una alta representatividad de las mismas en el ecosistema empresarial.

Por otro lado, otro de los argumentos que justifican la importancia de la figura de la microempresa en la actualidad es cómo contribuye ésta al desarrollo de la economía. En los cuadros 3 y 4 se exponen algunos datos que reflejan la importancia de las microempresas desde un punto de vista macroeconómico, en concreto a través de la aportación al empleo y al valor añadido de las economías española y europea, respectivamente.

La principal conclusión que se obtiene de los datos descriptivos expuestos en los cuadros 3 y 4 es que los más de veinte millones de pequeñas y medianas empresas registradas en la UE en 2010 y 2012 representaban el 99,8 por 100 del total de empresas, las cuales suponían más de dos tercios del empleo en Europa y casi el 60 por 100 del valor bruto de los activos. Por todo lo anterior, la pequeña y mediana empresa, y en concreto la microempresa, desempeñan un papel especialmente relevante en el desarrollo económico de la UE (Small Business Act First Implementation Report in Spain, 2009-2011; Annual Report on EU SMEs 2010/2011; Annual Report on European SMEs 2012/2013).

Siguiendo con la perspectiva económica, España ha pasado de crecer y crear empleo, con tasas de crecimiento de las mayores de Europa, a sufrir una fuerte desaceleración que ha derivado en la crisis económica más importante de España en las últimas décadas, provocando un impacto muy negativo en la creación de empleo y en la actividad empresarial del país. Según la Federación Nacional de Asociaciones de Trabajadores Autónomos, en España en el año 2009 , los trabajadores por cuenta propia representaban el 18,1 por 100 de los cotizantes.

\footnotetext{
${ }^{2}$ Datos del INE (consulta: 9 de febrero de 2014).
} 


\section{CUADRO 3: NÚMERO DE EMPRESAS, EMPLEO Y VALOR AÑADIDO BRUTO EN LA UE-27 POR TAMAÑO (2010)}

\begin{tabular}{|c|c|c|c|c|c|c|c|}
\hline & $\begin{array}{c}\text { Micro } \\
\mathbf{0 - 9}\end{array}$ & $\begin{array}{c}\text { Pequeñas } \\
\mathbf{1 0 - 4 9}\end{array}$ & $\begin{array}{c}\text { Medianas } \\
\mathbf{5 0 - 2 4 9}\end{array}$ & $\begin{array}{c}\text { PYME } \\
\mathbf{0 - 2 4 9}\end{array}$ & $\begin{array}{c}\text { Grandes } \\
\mathbf{2 5 0} \text { y más }\end{array}$ & Total \\
\hline \multicolumn{7}{|c|}{ Número de Empresas } \\
\hline Empresas & 19.198 .539 & 1.378 .401 & 219.252 & 20.796 .192 & 43.034 & 20.839 .226 \\
\hline$\%$ & 92,1 & 6,6 & 1,1 & 99,8 & 0,2 & 100 \\
\hline \multicolumn{7}{|c|}{ Empleo } \\
\hline Empleados & 38.905 .519 & 26.605 .166 & 21.950 .107 & 87.460 .792 & 43.257 .098 & 130.717 .890 \\
\hline$\%$ & 29,8 & 20,4 & 16,8 & 66,9 & 33,1 & 100 \\
\hline \multicolumn{7}{|c|}{ Valor Añadido Bruto } \\
\hline Millones $€$ & 1.293 .391 & 1.132 .202 & 1.067 .387 & 3.492 .979 & 2.485 .457 & 5.978 .436 \\
\hline$\%$ & 21,6 & 18,9 & 17,9 & 58,4 & 41,6 & 100 \\
\hline
\end{tabular}

Fuente: Eurostat/National Statistics Offices of Member States/Cambridge Econometrics/Ecorys en: Annual Report on European SMEs 2010/2011

\section{CUADRO 4: NÚMERO DE EMPRESAS, EMPLEO Y VALOR AÑADIDO BRUTO EN LA UE-27 POR TAMAÑO (2012)}

\begin{tabular}{|c|c|c|c|c|c|c|}
\hline & $\begin{array}{c}\text { Micro } \\
\mathbf{0 - 9}\end{array}$ & $\begin{array}{c}\text { Pequenas } \\
\mathbf{1 0 - 4 9}\end{array}$ & $\begin{array}{c}\text { Medianas } \\
\mathbf{5 0 - 2 4 9}\end{array}$ & $\begin{array}{c}\text { PYME } \\
\mathbf{0 - 2 4 9}\end{array}$ & $\begin{array}{c}\text { Grandes } \\
\mathbf{2 5 0} \text { y más }\end{array}$ & Total \\
\hline \multicolumn{7}{|c|}{ Número de Empresas } \\
\hline Empresas & 19.143 .521 & 1.357 .533 & 226.573 & 20.727 .627 & 43.654 & 20.771 .281 \\
\hline$\%$ & 92,2 & 6,5 & 1,1 & 99,8 & 0,2 & 100 \\
\hline \multicolumn{7}{|c|}{ Empleo } \\
\hline Empleados & 38.395 .819 & 26.771 .287 & 22.310 .205 & 87.477 .311 & 42.318 .854 & 129.796 .165 \\
\hline$\%$ & 29,6 & 20,6 & 17,2 & 67,4 & 32,6 & 100 \\
\hline \multicolumn{7}{|c|}{ Valor Añadido Bruto } \\
\hline Millones $€$ & $1.307 .360,7$ & $1.143 .935,7$ & $1.136 .243,5$ & 3.587 .540 & $2.591 .731,5$ & $6.179 .271,4$ \\
\hline$\%$ & 21,2 & 18,5 & 18,4 & 58,1 & 41,9 & 100 \\
\hline
\end{tabular}

Fuente: Eurostat/National Statistics Offices of Member States/Cambridge Econometrics/Ecorys en: Annual Report on European SMEs 2012/2013

A un nivel similar se encuentra Chipre (17,8 por 100), siendo superados estos porcentajes con creces en países como Grecia (30,2 por 100$)$, Italia (23,7 por 100$)$, Turquía (28,9 por 100) y Portugal (23,2 por 100). Las grandes empresas de banca, automoción y construcción crean empleo, pero las pequeñas lo hacen en mayor proporción, convirtiéndose en el motor de muchas de las economías de los países del sur de Europa. En este sentido, en el tercer trimestre de 2013 más de tres millones de personas se encontraban dadas de alta en el régimen especial de trabajadores autónomos, lo que representa más de un 18 por 100 del total de afiliados a la Seguridad Social y el 13 por 100 de los autónomos tienen empleados a su cargo, lo que supone un total de más de 800.000 empleos (INE 2014) ${ }^{3}$.

El tejido empresarial español, tal y como se desprende de los datos obtenidos de fuentes oficiales, es intensivo en pequeñas y medianas empresas, las cuales concentran gran parte del

${ }^{3}$ Datos obtenidos del tercer trimestre de 2013 del INE (INE 2014) 
empleo, al tiempo que presentan en promedio, bajos niveles de competitividad en comparación con la gran empresa (Platero-Jaime, 2014). En general, las PYMES españolas desarrollan su actividad en un círculo vicioso: dificultades para crear nuevos negocios, baja incentivación para pasar del autoempleo a economías de escala, barreras a la hora de abordar escenarios de comercio exterior, dificultades para disponer de crédito, limitaciones para capacitar a su talento o acceder a talento de alto valor, etc. Dicha situación da lugar a una alta mortalidad de las PYMES, una fuerte dependencia de los ciclos económicos y una baja propensión a crecer más allá del autoempleo y poder competir en mercados globales (Fundación Everis, 2010).

Ante un contexto económico y empresarial como el actual, la PYME parece jugar más que nunca un papel determinante en la reactivación de la economía española y europea (Fernández de Moya, 2013). Una posible vía para impulsar la recuperación económica podría ser construir un modelo productivo, basado en la innovación y el desarrollo tecnológico como factores claves para el aumento de la productividad y el empleo. La PYME juega por tanto un papel vital para canalizar las bases de dicha recuperación (Calleja, 2012). Parece relevante, por tanto, prestar especial atención al papel del empresario en el desarrollo de estrategias y a la toma de decisiones de dichas empresas, por lo que resulta especialmente interesante conocer el perfil de los empresarios en relación a sus aptitudes y conocimientos y cómo éstos pueden afectar a la capacidad innovadora de la empresa. Éste será uno de los grandes objetivos que se pretende estudiar en el presente trabajo, a partir del cual se espera obtener resultados que ayuden a comprender de una forma más clara y concluyente el funcionamiento del proceso innovador en la pequeña empresa y en concreto en el ámbito de la microempresa.

\section{EVOLUCIÓN DEL CONCEPTO "INNOVACIÓN". ADAPTACIÓN AL CONTEXTO DE LA MICROEMPRESA EN ESPAÑA}

El presente artículo pretende sentar las bases del significado del concepto "innovación" en la microempresa, analizando la evolución y adecuación del término en el contexto de la microempresa española. Por tanto, el presente trabajo se aleja de la perspectiva macroeconómica que han utilizado numerosos trabajos sobre la innovación en la empresa. A continuación se describen diferentes visiones y definiciones del concepto.

En términos generales, innovar se puede entender como el proceso a través del cual se introducen y aplican nuevas ideas y conocimientos (Rhee et al., 2010). En este sentido, se puede afirmar que la innovación se refiere a la creación de nuevos productos, servicios, procesos y métodos de organización, o adaptaciones de los existentes, sobre la base de nuevos conocimientos (Luecke y Katz, 2003; Wolf, 2006).

Así pues, la innovación puede ser entendida como la transformación de una idea en un producto nuevo o mejorado introducido en el mercado, o en un proceso operativo nuevo o mejorado utilizado en la industria o el comercio o en un nuevo método de servicio social (INE, 2000, p. 15).

En el contexto específico de la empresa, la innovación se lleva a cabo cuando se comercializa el conocimiento, por ejemplo, en forma de nuevos productos, servicios, procesos o modelos de negocio (Baldwin y Gellatly, 2003, Love et al., 2009). Amabile et al., (1996) define la innovación desde una perspectiva organizacional y la describe como la implementación exitosa de ideas creativas dentro de una organización. Según este enfoque, la creatividad de los individuos y los equipos son el punto de partida para la innovación. En esta línea, Gibson y Gibbs (2006) definen la innovación como un proceso colectivo de generación y aplicación de ideas que se basa en recursos, capacidades y personas de diferentes funciones organizativas. Andersson et al. (2008) definen la innovación como el conjunto de nuevas 
aplicaciones de conocimientos, ideas, métodos y habilidades que pueden generar capacidades únicas y aprovechar la competitividad de una organización.

En definitiva, innovar es un fenómeno complejo que implica producir, difundir y traspasar el conocimiento tecnológico para la creación de nuevos productos o procesos (Samara et al., 2012), y que trata de llevar a cabo un proceso de creación de nuevas ideas, que tengan valor como negocio o desde un punto de vista social (Morris, 2006).

La innovación supone la introducción de un nuevo, o significativamente mejorado, producto (bien o servicio), proceso, método de comercialización, o método organizativo bien en las prácticas internas de la empresa, en la organización del lugar del trabajo o en las relaciones exteriores (Manual de Oslo, 2005). Así, según Cilleruelo Carrasco et al. (2008) la innovación se podría definir como "el resultado original exitoso aplicable a cualquier ámbito de la sociedad, que supone un salto cuántico no incremental, y es fruto de la ejecución de un proceso no determinista que comienza con una idea y evoluciona por diferentes estadios; generación de conocimiento, invención, industrialización y comercialización, y que está apoyado en un paradigma organizacional favorable, en el que la tecnología supone un papel preponderante, y el contexto social en el que se valora la inversión en creación de conocimiento una condición necesaria".

El término innovación se ha definido en la literatura de diversas formas, dependiendo de la perspectiva adoptada por sus autores (OCDE, 2005; Jansen et al., 2006; Damanpour et al., 2009). No obstante, la mayoría de las definiciones propuestas inciden en que innovar implica adoptar una novedad para la empresa (Jiménez-Jiménez y Sánchez-Valle, 2013). En este sentido, por ejemplo, según García y Calantone (2002), innovar se puede entender como la generación o adopción de ideas, procesos, productos o servicios que la unidad relevante que los adopta los percibe como nuevos. Sin embargo, cuando se utiliza el término innovación en relación con el desarrollo de nuevos productos no siempre se hace desde una óptica interna, es decir, para aludir a las novedades o cambios que introduce la empresa en sus productos o línea de productos (Munuera y Rodríguez, 2007). Frecuentemente se define la innovación de productos desde una perspectiva externa o de mercado, esto es, como la introducción de productos que suponen alguna novedad para el mercado (García y Calantone, 2002).

Las anteriores definiciones de la innovación se pueden complementar con el concepto propuesto por Prieto Viñuela (2004). En este caso, el autor expone como la innovación es "el complejo proceso que lleva las ideas al mercado en forma de nuevos o mejorados productos o servicios", definición que no sería complementaria a las anteriores, de no ser por la descripción específica que hace del concepto estructurándolo en dos partes. Según el autor, una de las partes estaría especializada en el conocimiento y la otra estaría dedicada fundamentalmente a la aplicación del conocimiento para convertirlo en un proceso, un producto o un servicio que incorpore nuevas ventajas para el mercado y/o la sociedad.

Aunque existe gran variedad de definiciones del concepto "innovación" cada una de las cuales está enunciada según una perspectiva de estudio, conviene destacar cómo la definición que más se adapta al contexto específico de la pequeña empresa y en concreto de la microempresa, es la definición propuesta por García y Calantone (2002), ya que se exige como requisito conceptual el hecho de generar o adoptar ideas siendo éstas percibidas como nuevas por parte de la unidad que las genera o adopta. Precisamente, ante las limitaciones en términos de recursos que caracterizan la casuística de la pequeña empresa, la visión interna de innovar, a través de la percepción de adoptar ideas como nuevas en el día a día de la pequeña empresa refleja con mayor realismo el proceso innovador en el contexto de la microempresa y esta premisa es la base que contempla la presente investigación a la hora de considerar la innovación como concepto. 


\section{CUADRO 5: REVISIÓN DE LA DEFINICIÓN DEL CONCEPTO "INNOVACIÓN" SEGÚN LA LITERATURA}

\begin{tabular}{|c|c|}
\hline Fuente & Definición \\
\hline Amabile et al., (1996) & Implementación exitosa de ideas creativas dentro de una organización. \\
\hline $\operatorname{INE}(2000)$ & $\begin{array}{l}\text { Transformación de una idea en un producto nuevo o mejorado introducido } \\
\text { en el mercado, o en un proceso operativo nuevo o mejorado utilizado en la } \\
\text { industria o el comercio o en un nuevo método de servicio social }\end{array}$ \\
\hline $\begin{array}{l}\text { García y Calantone } \\
(2002)\end{array}$ & $\begin{array}{l}\text { Generación o adopción de ideas, procesos, productos o servicios que sean } \\
\text { percibidos como nuevos por la unidad relevante que los adopta, así como el } \\
\text { mercado. }\end{array}$ \\
\hline Luecke y Katz (2003) & $\begin{array}{l}\text { Creación de nuevos productos, servicios, procesos y métodos de } \\
\text { organización, o adaptaciones de los existentes, sobre la base de nuevos } \\
\text { conocimientos. }\end{array}$ \\
\hline Prieto Viñuela (2004) & $\begin{array}{l}\text { Complejo proceso que lleva las ideas al mercado en forma de nuevos o } \\
\text { mejorados productos o servicios, a través de la aplicación del conocimiento. }\end{array}$ \\
\hline (Manual de Oslo, 2005) & $\begin{array}{l}\text { Introducción de un nuevo, o significativamente mejorado, producto (bien o } \\
\text { servicio), proceso, método de comercialización, o método organizativo bien } \\
\text { en las prácticas internas de la empresa, en la organización del lugar del } \\
\text { trabajo o en las relaciones exteriores. }\end{array}$ \\
\hline $\begin{array}{l}\text { Gibson y Gibbs } \\
\text { (2006) }\end{array}$ & $\begin{array}{l}\text { Proceso colectivo de generación y aplicación de ideas que se basa en los } \\
\text { recursos, las capacidades y las personas de diferentes funciones } \\
\text { organizativas. }\end{array}$ \\
\hline (Morris, 2006) & $\begin{array}{l}\text { Proceso de creación de nuevas ideas, que tengan valor como negocio o } \\
\text { desde un punto de vista social. }\end{array}$ \\
\hline Andersson et al. (2008) & $\begin{array}{l}\text { Nuevas aplicaciones de conocimientos, ideas, métodos y habilidades que } \\
\text { pueden generar capacidades únicas y aprovechar la competitividad de una } \\
\text { organización. }\end{array}$ \\
\hline $\begin{array}{l}\text { Cilleruelo Carrasco et } \\
\text { al. (2008) }\end{array}$ & $\begin{array}{l}\text { Resultado original exitoso aplicable a cualquier ámbito de la sociedad, que } \\
\text { supone un salto cuántico no incremental, y es fruto de la ejecución de un } \\
\text { proceso no determinista que comienza con una idea y evoluciona por } \\
\text { diferentes estadios; generación de conocimiento, invención, } \\
\text { industrialización y comercialización, y que está apoyado en un paradigma } \\
\text { organizacional favorable, en el que la tecnología supone un papel } \\
\text { preponderante, y el contexto social en el que se valora la inversión en } \\
\text { creación de conocimiento una condición necesaria. }\end{array}$ \\
\hline Love et al. (2009) & $\begin{array}{l}\text { Comercialización del conocimiento en forma de nuevos productos, } \\
\text { servicios, procesos o modelos de negocio. }\end{array}$ \\
\hline Rhee et al. (2010). & Introducción y aplicación de nuevas ideas y conocimientos. \\
\hline Samara et al. (2012) & $\begin{array}{l}\text { Un fenómeno complejo que implica producir, difundir y traspasar el } \\
\text { conocimiento tecnológico para la creación de nuevos productos o procesos. }\end{array}$ \\
\hline $\begin{array}{l}\text { Jiménez-Jiménez } \quad y \\
\text { Sánchez-Valle (2013) }\end{array}$ & Adopción de una novedad para la empresa. \\
\hline
\end{tabular}

Fuente: Elaboración propia

En este sentido, la innovación también se podría definir como la tendencia de una empresa (Lumpkin y Dess, 1996) y la capacidad de ésta (Hult et al., 2004) para introducir innovaciones. En consecuencia, la capacidad de innovación se describe como la capacidad de introducir algún nuevo proceso, producto o idea en la organización (Hult et al., 2004) y refleja la tendencia de una empresa para participar y apoyar las nuevas ideas, la novedad, la experimentación y la procesos creativos que pueden dar lugar a nuevos productos, servicios o 
procesos tecnológicos (Lumpkin y Dess, 1996). En este sentido, Grant (2005) define una capacidad como lo que una empresa puede hacer, como producto de procesos de aprendizaje y conocimiento acumulado, de manera formal e informal, que tendrá como resultado un conjunto de procedimientos que buscan determinados objetivos (Barney, 1991; Peteraf, 1993). En el caso específico de la innovación, de acuerdo con Malaver y Vargas (2011), estas capacidades se entienden como las habilidades para mejorar significativamente o crear nuevos productos, procesos, métodos de marketing y organización. Hill y Neelly (2000) sugieren que la causa de la innovación en la empresa es su capacidad de innovación. En esta línea, Szeto (2000) define la capacidad de innovación como una mejora continua de las capacidades y recursos que la empresa posee con el fin de explorar y aprovechar las oportunidades para el desarrollo de nuevos productos para satisfacer las necesidades del mercado.

En definitiva, la capacidad de innovación a menudo se podría relacionar con las actividades formales e informales de I+D de la empresa, así como con los nuevos productos comercializados (Kirner et al., 2009). En este sentido, es necesario destacar cómo en los últimos años se ha puesto de manifiesto la existencia de una multiplicidad de formas de articulación de los procesos de innovación en las empresas (Buesa y Molero, 1998). Si bien es cierto que en la actualidad se reconoce el gran potencial innovador de las múltiples fuentes de aprendizaje incremental (por el uso, por la práctica, por el error, etc.), es innegable la importancia de las actividades de I+D como fuente que alimenta el proceso de innovación. En otras palabras, si bien en la actualidad se ha superado la visión que consideraba como única fuente de la innovación a las actividades de $\mathrm{I}+\mathrm{D}$, de tal forma que se asume que las actividades de innovación abarcan otras muchas además de las de $\mathrm{I}+\mathrm{D}$, lo cierto es que el potencial generador de innovaciones depende todavía en gran medida de la realización de estas actividades.

En suma, en el proceso de desarrollar una innovación intervienen toda clase de actividades científicas, tecnológicas, de organización, financieras y comerciales. La I+D es sólo una de esas actividades y puede ser realizada en diferentes etapas del proceso de innovación, siendo utilizada no sólo como fuente de ideas innovadoras sino también para resolver los problemas que pueden surgir en cualquier fase del proceso hasta su finalización (INE, 2000, p. 15).

En una primera aproximación, el concepto de I+D hace referencia al "proceso que permite obtener avances científicos de forma sostenida" (Cañibano, 1988, p. 35). Incluye la investigación básica, la investigación aplicada y el desarrollo experimental, abarcando "los trabajos creativos que se emprenden de modo sistemático a fin de aumentar el volumen de conocimientos, incluyendo el conocimiento del hombre, la cultura y la sociedad, así como la utilización de este volumen de conocimientos para concebir nuevas aplicaciones" (OCDE, 1981, p. 20).

La I+D debe diferenciarse de una amplia gama de actividades conexas que tienen una base científico-tecnológica. Estas actividades están relacionadas muy estrechamente con la I+D, tanto a través de los flujos de información como en lo concerniente a operaciones, instituciones y personal. En general, el criterio que permite distinguir la I+D de otras actividades conexas es la existencia, en el seno de la $\mathrm{I}+\mathrm{D}$, de un elemento apreciable de creatividad y resolución de una incertidumbre científica y/o tecnológica; o dicho de otra manera, cuando la solución de un problema no parezca evidente a cualquiera que esté al corriente del conjunto de conocimientos y técnicas básicas utilizadas comúnmente en el sector considerado (INE, 2000, p. 12).

En el ámbito de la microempresa se considera más habitual si cabe, la aplicación de los conocimientos con objeto de obtener un producto que termine siendo lanzado al mercado, ya 
que de esta forma, se consigue satisfacer al cliente y aumentar la cuota de mercado (Hooley et $a l .$, 2005). Generalmente, las innovaciones de productos se pueden manifestar en la mejora de los bienes o servicios existentes o en lugar de crear otros totalmente nuevos (Jansen et al., 2006). La innovación de productos resulta pues, crucial debido a su impacto en el desempeño de la organización (Atuahene-Gima, 1996) y, por lo tanto, sobre la supervivencia y el crecimiento de la empresa (Danneels, 2002). En esta línea, diversos estudios empíricos han demostrado los efectos positivos de la innovación de productos en el desempeño empresarial (Guan et al., 2009; Harmancioglu et al., 2010). Además, la innovación de productos refleja la capacidad de producción, de comercialización y de innovación (Chang y Cho, 2008; Dine Rabeh, 2014), posibilitando alcanzar la ventaja competitiva y asegurar el crecimiento en el largo plazo, mejorando el rendimiento empresarial (Dyer et al., 2009; Stock, 2011).

A pesar de que las innovaciones de productos y procesos son diferentes en cuanto a su origen, sus resultados y su visibilidad externa (Friedrich et al., 2010), ambas se encuentran estrechamente vinculadas, por lo que parece razonable que una medida adecuada del desempeño innovador debiera considerar los efectos tanto de la innovación de producto como de proceso (Prajogo y Ahmed, 2006). Sin embargo, en el caso de la microempresa parece coherente debido a su contexto, estudiar la innovación particularmente a través de la innovación de producto, ya que los cambios continuos que se producen en las necesidades de los clientes obligan a las empresas a desarrollar nuevos productos para mantener sus resultados y su posición competitiva en los mercados (Zhou, 2006; Tsai y Yang, 2013).

En esta línea Berends et al. (2013), sugieren la necesidad de continuar investigando sobre la innovación de producto en el contexto de la pequeña empresa y argumentan cómo:

- La mayoría de investigaciones en la gestión de la innovación de productos se ha centrado únicamente en las grandes empresas, y no han dejado de distinguir entre grandes y pequeñas empresas (Moultrie et al., 2007).

- Los estudios de innovación dirigidos específicamente a pequeñas firmas se han centrado en los antecedentes y consecuencias de los esfuerzos de innovación de producto (Brouwer y Kleinknecht, 1996; Ledwith y O'Dwyer, 2009), la identificación de los efectos de las colaboraciones inter-organizativas (Stuart, 2000; Bougrain y Haudeville, 2002); la orientación al competidor (Ledwith y O'Dwyer, 2009), la estructura organizativa (Terziovski, 2010), el capital intelectual (Leitner, 2011), y la disponibilidad de científicos e ingenieros cualificados (Romijn y Albaladejo, 2002).

\section{CONCLUSIONES Y FUTURAS LÍNEAS DE INVESTIGACIÓN}

La pequeña y mediana empresa, y en concreto la microempresa, desempeñan un papel especialmente relevante en el desarrollo económico de la Unión Europea, y especialmente en España. Por ello parece conveniente evaluar y estudiar en profundidad el concepto innovación en el ámbito específico de la microempresa.

Aunque existe gran variedad de definiciones del concepto "innovación" cada una de las cuales está enunciada según una perspectiva de estudio, conviene destacar cómo la definición que más se adapta al contexto específico de la pequeña empresa y en concreto de la microempresa, es la definición propuesta por García y Calantone (2002), ya que se exige como requisito conceptual el hecho de generar o adoptar ideas siendo éstas percibidas como nuevas por parte de la unidad que las genera o adopta.

Precisamente, ante las limitaciones en término de recursos que caracterizan la casuística de la pequeña empresa, la visión interna de innovar, a través de la percepción de adoptar ideas 
como nuevas en el día a día de la pequeña empresa refleja con mayor realismo el proceso innovador en el contexto de la microempresa y esta premisa es la base que contempla la presente investigación a la hora de considerar la innovación como concepto.

Además, la innovación es una actividad integral que implica a toda la organización (Iyer y Davenport, 2008; Dine Rabeh, 2014). Uno de los principales condicionantes de esta conducta es la capacidad innovadora (Clark y Ken, 1998; Fernández-Jardón, 2012), cuyo desarrollo exige la adaptación de la organización a las necesidades del proceso innovador (Marquardt, 1996; Martínez et al., 2011).

Por último, parece conveniente señalar cómo en el caso de las pequeñas y medianas empresas, la innovación de producto es más recurrida que la innovación de procesos, ya que las unidades empresariales de menor tamaño, parecen apostar por desarrollar productos específicos para nichos concretos del mercado, en lugar de entrar en mercados masivos (Vossen, 1998; Laforet, 2013).

Por todo lo anterior, resultaría deseable que futuros trabajos de investigación estudien y aborden la consideración de la innovación y sus formas, de manera que se puedan aportar nuevas contribuciones al estudio de la innovación en la pequeña empresa y en concreto en aquellas unidades empresariales que caracterizan el ecosistema empresarial español, como es el caso de la microempresas.

\section{BIBLIOGRAFÍA}

Amabile, T., Conti, R., Coon, H.,Lazenby, J. y Herron, M., (1996): "Assessing the work environment for creativity", Academy of Management Review, vol. 39, n 5, pp.11541184.

Andersson, A. y Lööf, H. (2011): "Small business innovation: firm level evidence from Sweden”, Small business innovation, no 37, pp. 732-754.

Andersson, M., Lindgren, R., y Henfridsson, O. (2008): "Architectural knowledge in interorganizational IT innovation”, Journal of Strategic Information Systems, vol. 17, n 1, pp. 19-38.

Andriopoulos, C., y Lewis, M.W. (2010): "Managing innovation paradoxes: Ambidexterity lessons from leading product design companies", Long Range Planning, vol. 43, $\mathrm{n}^{\circ} 1$, pp. 104-122.

Atuahene-Gima, K. (1996): "Differential potency of factors affecting innovation performance in manufacturing and services firms in Australia", Journal of Product Innovation, $\mathrm{n}^{\mathbf{0}}$ 13, pp. 35-52.

Audretsch, D. (2012): "Entrepreneurship research", Management Decision, vol. 50, n 5, pp. $755-764$.

Audretsch, D.B. y Lehmann, E.E. (2005): Mansfield's Innovation in the Theory of Innovation," in Essays in Honor of Edwin Mansfield-The Economics of $R \& D$, Innovation, and Technological Change, Eds. A. N. Link and F. M. Scherer, New York: Springer, pp. 281-290.

Baker, W.E., y Sinkula, J.M. (1999): "The synergistic effect of market orientation and learning orientation on organizational performance", Journal of Academy of Marketing Science, vol. 27, nº 4, pp. 411-427.

Baldwin, J. y Gellatly, G. (2003): Innovation strategies and performance in SMEs, Cheltenham, UK: Edward Elgar.

Barney, J. (1991): "Firm resources and sustained competitive advantage", Journal of Management, vol. 17, $\mathrm{n}^{\mathrm{o}}$ 1, pp. 99-120. 
Bednarzik, R. W. (2000): "The role of entrepreneurship in U.S. and European job growth", Monthly Labor Review, vol. 123, nº 7, pp. 3 - 16.

Berends, H., Jelinek, M, Reymen, I. y Stultiëns, R. (2013): "Product innovation processes in small firms: Combining entrepreneurial effectuation and managerial causation", Journal of Product Innovation Management, DOI: 10.1111/jpim.12117

Bhaskaran, S. (2006): "Incremental innovation and business performance: Small and mediumsize food enterprises in a concentrated industry environment", Journal of Small Business Management, vol. 44, $\mathrm{n}^{\circ}$ 1, pp. 64-80.

Booyens, I. (2011): "Are small, medium- and micro-sized enterprises engines of innovation? The reality in South Africa", Science and Public Policy, vol. 38, n 1, pp. 67-78.

Bougrain, F. y Haudeville, B. (2002): "Innovation, collaboration and SMEs' internal research capacities", Research Policy, no 31, pp. 735-47.

Boumediene, R., Lorenzo, O. y Kawalek, P. (2009): "Information systems innovations adoption and diffusion among SMEs: Current status and future prospects", International Journal of Eadoption, $\mathrm{n}^{\mathrm{o}}$ 1, pp. 33-45.

Broekel, T. y Brenner, T. (2011): "Regional factors and innovativeness: an empirical analysis of four German industries", Ann Reg Sci, n 47, pp. 169-194

Brouwer, E. y Kleinknecht, A. (1996): "Firm size, small business presence and sales of innovative products: a micro-econometric analysis", Small Business Economics, $\mathrm{n}^{\circ}$ 8, pp. 1898-1201.

Buesa, M. y Molero, J. (1998): "La regularidad innovadora en empresas españolas", Revista de Economía Aplicada, vol. 6, nº 17, pp 111-134.

Calleja, D. (2012): Fundación para el Desarrollo Infotecnológico de Empresas y Sociedad. Protragonistas, 9 de Abril de 2012: http://www.fundetec.es/protagonistas/4579/

Camisón, C., y Villar-López, A. (2014): "Organizational innovation as an enabler of technological innovation capabilities and firm performance", Journal of Business Research, vol. 67, n 1, pp. 2891-2902.

Cañibano, L. (1988): Costes de Investigación y Desarrollo, Instituto de Contabilidad y Auditoría de Cuentas. Ministerio de Economía y Hacienda, Madrid.

Chang, D. R. y Cho, H. (2008): “Organizational memory influences new product success", Journal of Business Research, vol. 61, n 1, pp. 13-23.

Cilleruelo Carrasco, E., Sánchez Fuente, F., y Etxebarría Robledo, B. (2008): “A compendium of definitions of the «innovation» concept by relevant authors: An up-todate hybrid design of the concept", Dirección y Organización, $\mathrm{n}^{\circ} 36$.

Clark, J. y Ken, G. (1998): "Innovation and competitiveness: A review”, Technology Analysis \& Strategic Management, vol. 10, $\mathrm{n}^{\circ}$ 3, pp. 363-395.

Comisión Europea (2003): (Texto pertinente a efectos del EEE) (2003/361/CE). En: Diario Oficial de la Unión Europea, 20 de mayo de 2003.

Comisión Europea (2008): "Think small first. A "small business act" for Europe". Communication from the Commission to the Council, the European Parliament, the European Economic and Social Committee and the Committee of the regions. Bruselas 25.6.2008 COM(2008), p. 394 final

Comisión Europea (2013a): Performance of SMEs in 2012: amidst challenges, prospects improving for most Member States, MEMO 12 / 1045. Brussels, 26 November. 
Comisión Europea (2013b): A recovery on the horizon?, Final report. Annual report on European SMEs 2012/2013, Octubre.

Damanpour, F., Walter, R.M. y Avellaneda, C.N. (2009): "Combinative effects of innovation types and organizational performance: A longitudinal study of service organizations", Journal of Management Studies, vol. 46, nº 4, pp. 650-675.

Danneels, E. (2002): "The dynamics of product innovation and firm competences", Strategic Management Journal, 23 (12), pp. 1095-1121.

Dine Rabeh, H.A. (2014): "Organizational capabilities for innovation development: An empirical approach", Degree of Ph.D. in Business Sciences. Universidad de Murcia.

Dirección General de Política de la Pequeña y Mediana Empresa (2011): Small Business Act First implementation report in Spain, 2009-2011, NIPO: 701-11-040-0. ECPMITYC: 1. ${ }^{\mathrm{a}} \mathrm{Ed} . / 0711$

Dyer, J.H., Gregersen, H.B. y Christensen, C.M. (2009): “The innovator's DNA”, Harvard Business Review, vol. 87, n 12, pp. 60-67.

Fernández de Moya, J.E. (2013): "Las pymes, el motor de la economía", Agenda de la empresa andaluza: ideas, personas e instrumentos para dirigir la empresa, $\mathrm{n}^{\mathrm{o}} 182, \mathrm{p}$. 9.

Fernández-Jardón, C.M. (2012): “Determinantes de la capacidad de innovación en PYMES regionales", Documentos de traballo do Departamento de Economía Aplicada, $\mathrm{n}^{\mathrm{o}} 2$, pp. 1-21.

Friedrich, T.L., Mumford, M.D., Vessey, B., Beeler, C.K. y Eubanks, D.L. (2010): "Leading for innovation. International”, Studies of Management and Organization, vol. 40, n⿳2 2 , pp. 6-29.

Fundación Everis (2010): Un momento clave de oportunidad para construir entre todos la España admirada del futuro, Disponible en: www.larazon.es/documents/get_document/43502.

García, R. y Calantone, R. (2002): "A critical look at technological innovation typology and innovativeness terminology: A literature review", Journal of Product Innovation Management, $\mathrm{n}^{\mathrm{o}}$ 19, pp. 110-132.

Gibson, C.B., y Gibbs, J.L. (2006): "Unpacking the concept of virtuality: The effects of geographic dispersion, electronic dependence, dynamic structure, and national diversity on team innovation", Administrative Science Quarterly, vol. 51, $\mathrm{n}^{\mathrm{o}} 3$, pp. 451-495.

Grant, R. (2005): Contemporary Strategy Analysis. Oxford: $5^{\text {th }}$ ed., Blackwell.

Guan, J.C., Yam, R.C.M., Tang, E.P.Y. y Lau, A.K.W. (2009): "Innovation strategy and performance during economic transition: Evidences in Beijing, China", Research Policy, vol. 38, n 5 , pp. 802-812.

Güngör, Ö.D. y Gözlu, S. (2012): "Influencing factors of innovation for Turkish companies", International Journal of Quality and Service Sciences, vol. 4, n 4, pp. 374-386

Hamel, G. (2000): Leading the revolution, Harvard Business School Press, Boston, MA.

Harmancioglu, N., Grinstein, B. y Goldman, A. (2010): "Innovation and performance outcomes of market information collection efforts: The role of top management team involvement", International Journal of Research in Marketing, vol. 27, nº 1 , pp. 3343. 
Hashi, I., y Stojčić, N. (2013): “The impact of innovation activities on firm performance using a multi-stage model: Evidence from the community innovation survey 4", Research Policy, vol. 42, n' 2, pp. 353-366.

Hill, J., y Neely, N. (2000): "Innovative capacity of firms: on why some firms are more innovative than others", $7^{\text {th }}$ International Annual EurOMA Conference 2000. Ghent.

Hitt, M.A., Ireland, R.D., Clifford, P.G. y Coyne, K.P. (2001): “Guest editors' introduction to the special issue strategic entrepreneurship: Entrepreneurial strategies for wealth creation", Strategic Management Journal, n 22, pp. 479-491.

Hooley, G.J., Greenley, G.E., Cadogan, J. W. y Fahy, J. (2005): “The performance impact of marketing resources", Journal of Business Research, no 58(Special Issue), pp. 18-27.

Hult, G.T.M., Hurley, R.F. y Knight, G.A. (2004): "Innovativeness: its antecedents and impact on business performance", Industrial Marketing Management, vol. 33, $\mathrm{n}^{\circ}$ 5, pp. 429-438.

Hutter, K., Hautz, J., Repke, K. y Matzler, K. (2013): "Open innovation in small and micro enterprise", Problems and Perspectives in Management, vol. 11, $\mathrm{n}^{\mathrm{o}}$ 1, pp. 12-22.

Instituto Nacional de Estadística (INE) (2000): Estadística sobre las Actividades en Investigación Científica y Desarrollo Tecnológico (I+D), Instituto Nacional de Estadística, Madrid.

Instituto Nacional de Estadítica (2013): Directorio Central de Empresas (DIRCE)

Iyer, B. y Davenport, T. H. (2008): "Reverse engineering Google's innovation machine", Harvard Business Review, vol. 86, n 4, 58-68.

Jansen, J. J. P., Van Den Bosch, F. A. J. y Volberda, H. W., (2006): "Exploratory innovation, exploitative innovation, and performance: Effects of organizational antecedents and environmental moderators", Management Science, vol. 52, nº 11, pp. 1661-1674.

Jiménez Jiménez, D. y Sanz Valle, R. (2013): "Studying the effect of HRM practices on the knowledge management process", Personnel Review, vol. 42, n 1, pp. 28-49. DOI: $10.1108 / 00483481311285219$.

Kirner, E., Kinkel, S. y Jaeger, A. (2009): "Innovation paths and the innovation performance of low-technology firms - an empirical analysis of German industry", Research Policy, vol. 38, n⿳3 3, pp. 447-458.

Kmieciak, R., Michna, A. y Meczynska, A. (2012): "Innovativeness, empowerment and IT capability: evidence from SMEs”, Industrial Management \& Data Systems, vol. 112, $\mathrm{n}^{\circ} 5$, pp. $707-728$

Koski, H., Marengo, L. y Mäkinen, I. (2009): "Firm size, managerial practices and innovativeness: Some evidence from Finnish manufacturing", LEM Working Paper Series, No. 2009/01.

Kunz, W., Schmitt, B. y Meyer, A. (2011): "How does perceived firm innovativeness affect the consumer?", Journal of Business Research, n 64, pp. 816-822.

Laforet, S. (2013): "Organizational innovation outcomes in SMEs: Effects of age, size, and sector”, Journal of World Business, $\mathrm{n}^{\circ}$ 48, pp. 490-502.

Ledwith, A. y O’Dwyer, M. (2009): “Market orientation, NPD performance, and organizational performance in small firms", Journal of Product Innovation Management, 26(6), 652-661.

Leitner, K.H. (2011): "The effect of intellectual capital on product innovativeness in SMEs", International Journal of Technology Management, vol. 53, $\mathrm{n}^{\mathrm{o}}$ 1, pp. 1-18. 
Love, J.H. y Roper, S. (2001): "Location and network effects on innovation success: evidence for UK, German and Irish manufacturing plants", Research Policy, vol. 30, n 4, pp. 64361.

Love, J.H., S. Roper, y Du, J. (2009): "Innovation, Ownership and Profitability", International Journal of Industrial Organization, $\mathrm{n}^{\circ} 27$, pp. 424- 434.

Luecke, R. y Katz, R. (2003): Managing creativity and innovation, Harvard Business School Press, MA.

Lumpkin, G.T. y Dess, G.G. (1996): "Clarifying the entrepreneurial orientation construct and linking it to performance", Academy of Management Journal, vol. 21, $\mathrm{n}^{\mathrm{o}} 1$, pp. 13572.

Malaver, F. y Vargas, M. (2011): Formas de innovar, desempeño innovador y competitividad industrial. Un Estudio a partir de la Segunda Encuesta de Innovación en la Industria de Bogotá y Cundinamarca, Bogotá, Colombia: Editorial Pontificia Universidad Javeriana: Cámara de Comercio de Bogotá.

Marquardt, M.J. (1996): Building the learning organization.a system approach to quantum improvement and global success, New York: McGraw-Hill.

Martínez, J.A., Gamero, J. y Tamayo, J.A. (2011): "Hacia una tipología empresarial basada en los factores explicativos de la intensidad innovadora: una aplicación empírica", Revista Europea de Dirección y Economía de la Empresa, vol. 20, nº1, pp. 143-160

Mogollón, R. y Vaquero, A. (2004): "El comportamiento innovador y los resultados de la empresa: Un análisis empírico", Proceedings of the XVIII Congreso Anual y XIV Congreso Hispano-Francês, AEDEM, Ourense, Spain.

Morales, M.E., Ortíz Riaga, C. y Arias Cante, M.A. (2013): "Factores determinantes de los procesos de innovación: una mirada a la situación en Latinoamérica", Rev. Esc.adm.neg., $\mathrm{n}^{\mathrm{o}}$ 72, pp. 148-163

Morris, L. (2006): Permanent innovation (1 ed.), Walnut Creek CA, USA: Innovation Academy.

Moultrie, J., Clarkson, P.J. y Probert, D. (2007): "Development of a design audit tool for SMEs", Journal of Product Innovation Management, vol. 24, n 4, pp. 335-368.

Munuera, J.L. y Rodríguez, A.I. (2007): Estrategias de marketing: Un enfoque basado en el proceso de dirección, Madrid: ESIC.

Nieto, M.J. y Santamaría, L. (2010): "Technological collaboration: Bridging the innovation gap between small and large firms", Journal of Small Business Management, $\mathrm{n}^{\circ} 48$, pp. 44-69.

OCDE (1981): La Medición de las Actividades Científicas y Técnicas: Manual de Frascati, Centro para el Desarrollo Tecnológico Industrial (CDTI), Madrid.

OECD (2005): Oslo Manual: Guidelines for collecting and interpreting innovation, $3^{\text {rd }}$. Edition. Paris, OECD Publications.

Peteraf, M.A. (1993): "The cornerstones of the competitive advantage: A resource -based View", Strategy Management Journal, n 14, pp. 179-192.

Platero-Jaime, M. (2014): “La innovación en la empresa española: ¿el tamaño importa?", Revista APD: Asociación para el Progreso de la Dirección, ISSN 1886-1709, nº. 294, 2014 (Ejemplar dedicado a: Innovación), pp. 28-31

Porter, M.E. (1990): The Competitive Advantage of Nations, The Free Press, New York.

Porter, M.E. (1996): “What is strategy?", Harvard Business Review, Nov.-Dec., pp. 60-80. 
Prajogo, D.I. y Ahmed, P.K. (2006): "Relationships between innovation stimulus, innovation capacity, and innovation performance", $R \& D$ Management, $\mathrm{n}^{\circ} 36$, pp. 499-515.

Prieto Viñuela, J.J. (2004): “Competitividad e innovación tecnológica. El sistema español de innovación", IX Jornadas de Economía Crítica, Disponible en PDF www.ucm.es/info/ec/jec9/pdf/A11\%20-\%20Prieto,\%20Juan\%20José.pdf.

Rhee, J., Park, T. y Lee, D.H. (2010): "Drivers of innovativeness and performance for innovative SMEs in South Korea: Mediation of learning orientation", Technovation, $30,65-75$

Roberts, P. y Amit, R. (2003): "The dynamics of innovative activity and competitive advantage: The case of Australian retail banking, 1981 to 1995", Organization Science, vol. 14, n 2, pp. 107-122.

Romero, I. y Martínez-Román, J.A. (2012): "Self-employment and innovation. Exploring the determinants of innovative behavior in small businesses", Research Policy, no 41, pp. $178-189$.

Romijn, H. y Albaladejo, M. (2002): "Determinants of innovation capability in small electronics and software firms in southeast England", Research Policy, $\mathrm{n}^{\mathbf{o}}$ 31, pp. $1053-1067$.

Rowley, J., Baregheh, A. y Sambrook, S. (2011): "Towards an innovation-type mapping tool", Management Decision, vol. 49, n' 1, pp. 73-86.

Samara, E., Georgiadis, P., y Bakouros, I. (2012): "The impact of innovation policies on the performance of national innovation systems: A system dynamics analysis", Technovation, vol. 32, $\mathrm{n}^{\mathrm{o}}$ 11, pp. 624-638.

Schumpeter, J.A. (1934): The theory of economic development, Harvard University Press, Cambridge: MA.

Simon, H. (2009): Hidden champions of the twenty-first century. The success strategies of unknown world market leaders, Springer, New York, NY.

Stock, R.M. (2011): "How does product program innovativeness affect customer satisfaction? A comparison of goods and services", Journal of the Academy of Marketing Science, vol. 39, nº 6, pp. 813-827.

Stuart, T.E. (2000): "Interorganizational alliances and the performance of firms: A study of growth and innovation rates in a high-technology industry", Strategic Management Journal, vol. 21, no 8, pp. 791-811.

Szeto, E. (2000): "Innovation capacity: working towards a mechanism for improving innovation within an inter-organizational network", The TQM Magazine, vol. 12, $\mathrm{n}^{\circ}$ 2, pp. 149-158.

Terziovski, M. (2010): "Innovation practice and its performance implications in small and medium enterprises (SMEs) in the manufacturing sector: a resource-based view", Strategic Management Journal, vol. 31, nº 8, pp. 892-902.

Tsai, Kuen - Hung y Yang, Shu - Yi. (2013): "Firm innovativeness and business performance: The joint moderating effects of market turbulence and competition", Industrial Marketing Management, $\mathrm{n}^{\mathrm{o}}$ 42, pp. 1279-1294.

Vossen, R. (1998): "Relative strengths and weaknesses of small firms in innovation", International Small Business Journal, vol. 16, n 3, pp. 88-94.

Wennekers, S., Van Stel, A., Thurik, R., y Reynolds, P. (2005): "Nascent entrepreneurship and the level of economic development", Small Business Economics, vol. 24, n 3, pp. 293-309. 
Wolf, S. (2006): "Encouraging innovation and productivity growth in Africa to create decent jobs. DPRU/TIPS, Conference on Accelerated and Shared Growth in South Africa: Determinants, Constraints and Opportunities" 18-20 October 2006, Johannesburg, South Africa.

Zahra, S., Belardino, S. y Boxx, W. (1988): "Organizational innovation: Its correlates and its implications for financial performance", International Journal of Management, $\mathrm{n}^{\mathrm{o}}$ 5, pp. $133-142$.

Zhou, K.Z. (2006): "Innovation, imitation, and new product performance: The case of China", Industrial Marketing Management, vol. 35, nº 3, pp. 394-402. 